\title{
Evaluation of GPS/BeiDou Integration Positioning Performance*
}

\author{
Yun ZHANG, ${ }^{1)}$ Tiantian Wu, ${ }^{1,2)}$ Yongming LiU, ${ }^{2)}$ Yanling HAN, ${ }^{1)}$ Chunming FAN, ${ }^{3)}$ and Akio YASUDA ${ }^{3)}$ \\ ${ }^{1)}$ Shanghai Ocean University, Shanghai 201306, China \\ ${ }^{2}$ Shanghai Maritime University, Shanghai 201306, China \\ ${ }^{3)}$ Tokyo University of Marine Science and Technology, Tokyo 108-8477, Japan
}

\begin{abstract}
As a new global navigation system, BeiDou has formally been in operation in the Asian region since December 27, 2012. To evaluate its positioning performance, several days' observation data were collected using a global positioning system (GPS)/BeiDou receiver in Shanghai. In this paper, the constellation and signals of BeiDou are first reviewed. Carrier-to-noise density ratio $\left(\mathrm{C} / \mathrm{N}_{0}\right)$ and multipath combinations are analyzed, then multipath errors of GPS satellites and BeiDou satellites were compared in terms of elevation angle. A positioning performance comparison of three scenarios was conducted using three indicators including number of visible satellites (NVS), position dilution of precision (PDOP), and accuracy of positioning. All positioning results were solved using a single-point positioning mode. After six months of adjustment, comparison of the positioning performance before BeiDou began providing regional services, there were some significant improvements in performance. The accuracy requirements published before formal operation, in which positioning accuracy is less than $10 \mathrm{~m}$, was met. The results show that the integration system has a large NVS, low PDOP value, and a high level of accuracy and precision. The positioning performance can be improved after using BeiDou, especially under the conditions of urban environment.
\end{abstract}

Key Words: GPS, BeiDou, Positioning Performance, Urban Environment

\section{Introduction}

China is now establishing its own global navigation satellite system known as BeiDou-2. Following the successful development and operation of the BeiDou Satellite Navigation Experimental System (BeiDou-1), the BeiDou-2 system was initiated in 2007 with the launch of the first navigation satellite into a medium-altitude earth orbit. ${ }^{1,2)}$ The interface control document (ICD) describing the details of the BeiDou B1I open service signal at $1561.098 \mathrm{MHz}$ was released by the Chinese State Council Information Office on December 27, 2012 at a news conference held in Beijing. ${ }^{3)}$ This means that the BeiDou system is formally in operation in the Asian region. In past studies on BeiDou, the investigation mainly focused on satellite visibility, validity of measurements, carrier-to-noise density ratio $\left(\mathrm{C} / \mathrm{N}_{0}\right)$, code noise and positioning results analysis. ${ }^{4-8)}$ Extensive research has also been conducted on position dilution of precision (PDOP) ${ }^{9-13)}$ Multipath error seriously affects the precision in high-precision GPS applications, and some articles have analyzed multipath error and detection methods. ${ }^{14,15)}$ The analysis of combined GPS/BeiDou data quality and positioning accuracy under different observation conditions has also been proposed. ${ }^{16)}$

Based on the above-mentioned studies, this study further evaluates the signal performance of the BeiDou MEO/ IGSO/GEO satellite, and BeiDou positioning performance during two different periods (before and after formal BeiDou regional operation). Positioning performance after integrat-

(C) 2015 The Japan Society for Aeronautical and Space Sciences

*Received 6 November 2013; final revision received 15 November 2014; accepted for publication 19 November 2014. ing GPS and BeiDou is also discussed in this study. We also carried out an experiment in an urban environment. The positioning performance after using BeiDou was evaluated.

\section{Data Collection}

The BeiDou navigation system is an independent global satellite navigation system. The final constellation will have 35 satellites consisting of 5 geostationary (GEO) satellites, 3 inclined geosynchronous (IGSO) satellites and 27 medium earth orbit (MEO) satellites. With the rapid development of China's space technology, construction of the BeiDou navigation system has basically been completed including GEO satellites and IGSO satellites, and there are 4 MEO satellites currently orbiting in space. BeiDou GEO satellites C01, C02, C03, C04 and C05 were launched on January 17, 2010, October 25, 2012, June 6, 2010, November 1, 2010 and February 25,2012 , respectively. Their tracks are fixed, projected over the equator and respectively located at $140^{\circ} \mathrm{E}, 80^{\circ} \mathrm{E}$, $110.5^{\circ} \mathrm{E}, 160^{\circ} \mathrm{E}$ and $58.75^{\circ} \mathrm{E}$. Five IGSO satellites, BeiDou-C06, C07, C08, C09 and C10, were launched on August 1, 2010, December 18, 2010, April 10, 2011, July 27, 2011 and December 2, 2011, respectively. Five MEO satellites were launched on April 14, 2007, April 30, 2012 and September 19, 2012, respectively. As the ICD stipulated, the BeiDou system will be established completely and provide global service by 2020 . The whole constellation structure is shown in Fig. 1.

The BeiDou timing system (BDT) starts from UTC 00:00:00, January 1, 2006, which is 14-second difference from GPS time $($ GPST), i.e. GPST $=$ BDT $+14 \mathrm{~s}$. The two timing systems have a system clock bias in the process of estimation receiver position, and the Chinese Geodetic 


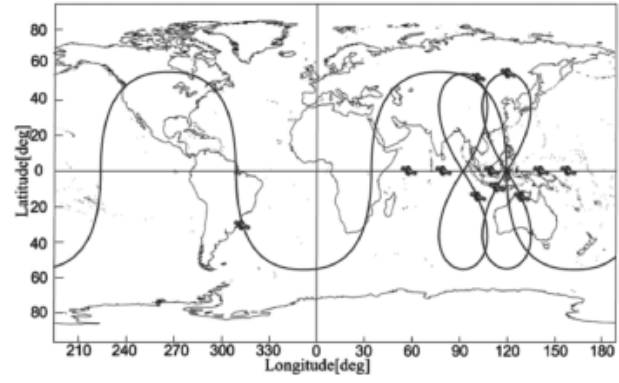

Fig. 1. Footprint of the BeiDou constellation

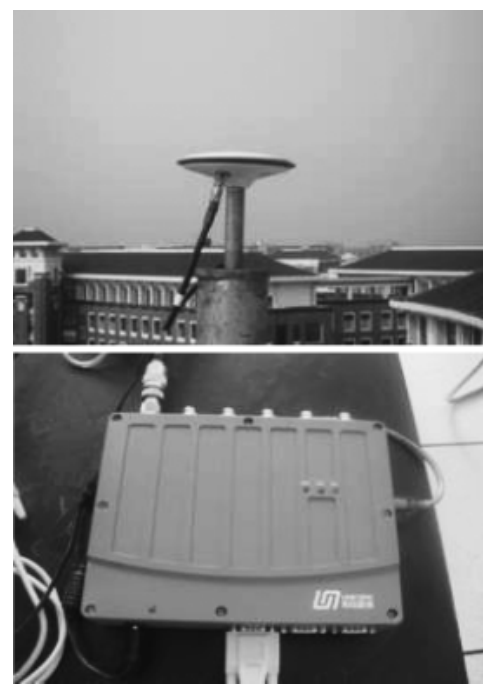

Fig. 2. Antenna of receiver located on the roof of a building at Shanghai Ocean University (top), GPS/BeiDou receiver (bottom).

Coordinate System is used in the BeiDou system. In the experiment, we received two frequencies centered at 1561.089 MHz (B1) and 1207.14 MHz (B2) from BeiDou, and two other frequencies centered at $1575.42 \mathrm{MHz}$ (L1) and $1227.6 \mathrm{MHz}$ (L2) from a GPS.

Figure 2 displays the antenna and receiver used in this study. The UR240-CORS GPS/BeiDou quad-frequency, high-precision receiver was selected for this experiment. It can receive BeiDou B1/B2 and GPS L1/L2 signals. The UA240-CP GPS/BeiDou quad-frequency high-gain antenna supports this receiver. The antenna is located on the roof of the College of Information Technology. In order to observe the multipath variations with elevation changes, we set the mask angle at 5 degrees.

Table 1 lists the information about the equipment in detail. The frequencies of GPS and BeiDou are also listed respectively, and the output rate of positioning data is set at $1 \mathrm{~Hz}$. Table 2 displays the time of the observation data that BeiDou has provided since beginning formal regional operation in December 2012. Several days' observation data were collected for this research in July 2012 (before formal regional operation) and January 2013 (after formal regional operation).

Figure 3 (top) shows the BeiDou navigation system sky plot for 24 hours on July 17, 2012 as observed in Shanghai.
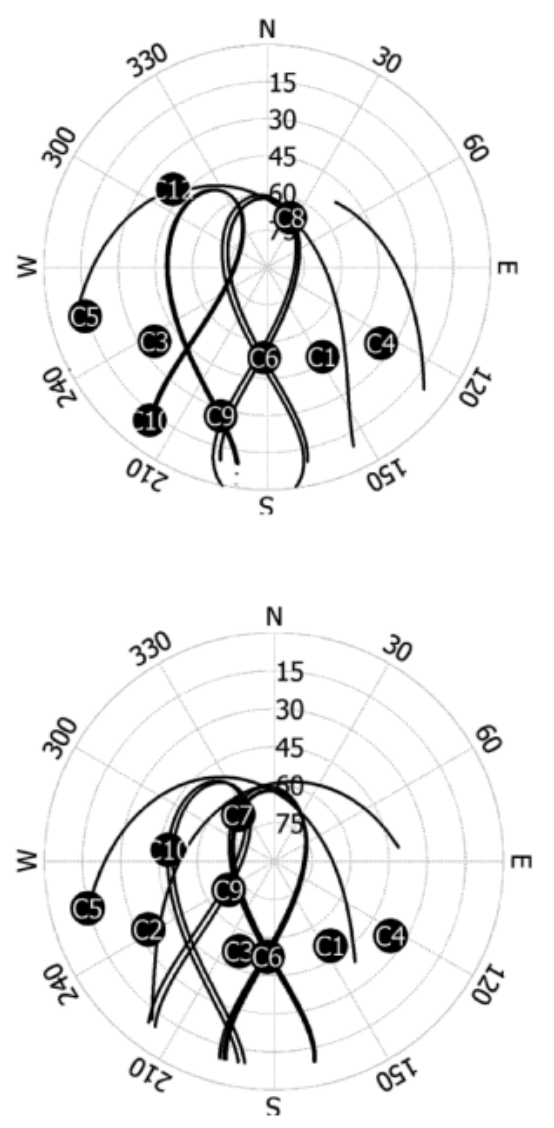

Fig. 3. The skyplot of the BeiDou navigation system for 24 hours on July 17, 2012 (top) and January 21, 2013 (bottom) in Shanghai.

Table 1. Equipment configuration and signal information.

\begin{tabular}{ccllrr}
\hline Receiver & Antenna & & \multicolumn{2}{c}{ Signal (MHz) } & Output \\
\hline UR240-CORS & UA240-CP & GPS: & L1:1575.42, L2:1227.60 & $1 \mathrm{~Hz}$ \\
& & BeiDou: B1:1561.089, B2:1207.14 & \\
\hline
\end{tabular}

Table 2. Test date for observation data and space constellation.

\begin{tabular}{lcc}
\hline & $\begin{array}{c}\text { Before BeiDou formal } \\
\text { regional operation }\end{array}$ & $\begin{array}{c}\text { After BeiDou formal } \\
\text { regional operation }\end{array}$ \\
\hline Day 1 & $2012-7-17$ & $2013-1-21$ \\
Day 2 & $2012-7-19$ & $2013-1-23$ \\
\hline \multicolumn{2}{c}{ Space constellation } \\
\hline BeiDou & 4GEO+5IGSO+2MEO 5GEO+5IGSO+4MEO \\
Mask angle & 5 degrees \\
Results & NVS, PDOP, accuracy of single point position \\
\hline
\end{tabular}

GEO satellites C01, C03, C04 and C05 have the stationary track in this figure. IGSO satellites C06, C07, C08, C09 and $\mathrm{C} 10$ have a repeated figure-8-shaped ground track. In addition, $\mathrm{C} 09$ and $\mathrm{C} 10$ in the same track are different from C06, C07 and C08. Figure 3 (bottom) shows the BeiDou navigation system sky plot for 24 hours observed on January 21 , 2013. GEO satellites locations have changed some as compared to Fig. 3 (top). C02 appeared and replaced the location of $\mathrm{C} 03$. C03 moved eastward and is located at the center of five GEO satellites. 


\section{Quality Analysis of GPS/BeiDou Measurements}

In this section, the quality of the acquired data is analyzed in five aspects; namely $\mathrm{C} / \mathrm{N}_{0}$, multipath error, NVS, PDOP and accuracy of position. Finally, data collected in urban environment is also analyzed.

The $\mathrm{C} / \mathrm{N}_{0}$ is defined as a signal to noise ratio, and it is related to receiver, signal propagation path and satellite observation elevations. As early as 2000, Satirapod and Wang began to see $\mathrm{C} / \mathrm{N}_{0}$ and elevations as standards to measure the effectiveness of GPS observations. ${ }^{17)}$

The multipath errors were computed using the method introduced by Kee and Parkinson in the paper for calibrating multipath errors on pseudorange measurements. ${ }^{18-20)}$ The pseudorange and carrier phase measurements are shown as follows.

$$
\begin{aligned}
& \rho=r+c\left(\delta_{t}-\delta^{k}\right)+T+I+M_{\rho}+\varepsilon_{\rho} \\
& \Phi=r+c\left(\delta_{t}-\delta^{k}\right)+T-I+M_{\Phi}+N+\varepsilon_{\Phi}
\end{aligned}
$$

where $\rho$ and $\Phi$ represent the observable code range and carrier phase, respectively, $r$ is the geometric satellite receiver distance, $c$ is the speed of light, $\delta_{t}$ and $\delta^{k}$ represent the receiver clock offset and satellite clock offset, respectively, $T$ is the neutral atmospheric delay, $I$ is the ionospheric delay, $N$ is the carrier phase ambiguity, $M_{\rho}$ and $\varepsilon_{\rho}$ are the multipath error and random noise of pseudorange measurements, and $M_{\Phi}$ and $\varepsilon_{\Phi}$ are the multipath error and random noise of carrier phase measurements.

Because $M_{\rho} \gg M_{\Phi}$ and $\varepsilon_{\rho} \gg \varepsilon_{\Phi}$, using Eq. (1) minus Eq. (2) with dual-frequency pseudorange and carrier phase measurement, assuming that the receiver can get continuous measurements and no cycle slip occurs,we can get:

$$
\begin{aligned}
M_{1} & =\rho_{1}-(\alpha+1) \cdot \Phi_{1}+\alpha \cdot \Phi_{2} \\
M_{2} & =\rho_{2}-(\beta+1) \cdot \Phi_{2}+\beta \cdot \Phi_{1} \\
\alpha & =2 \cdot f_{2}^{2} /\left(f_{1}^{2}-f_{2}^{2}\right) \\
\beta & =2 \cdot f_{1}^{2} /\left(f_{2}^{2}-f_{1}^{2}\right)
\end{aligned}
$$

where $M_{1}$ and $M_{2}$ are the code multipath error of the signal B1/L1 and B2/L2, $\rho_{2}$ and $\Phi_{1}$ are pseudorange and carrier phase of signal B1/L1, and $\rho_{1}$ and $\Phi_{2}$ are pseudorange and carrier phase of signal B2/L2. The frequencies we used are $f_{1}$ and $f_{2}$. The combination eliminates all error spread between satellites and receiver except for receiver noise, multipath error and bias variation from the pseudorange observation.

It's noteworthy that $M_{1}$ (or $M_{2}$ ) not only includes the multipath error of B1/L1 (or B2/L2) pseudorange measurements, but also includes carrier phase ambiguity, random noise and a hardware bias combination. In order to delete the carrier phase ambiguity, continuous measurements are necessary here.

The positioning function can be used only when signals are received from at least four visible satellites. In this way, the solution can be found since there were more equations than unknowns in a set of equations. So the number of effectively visible satellites is an important sign of the effectiveness of the system. ${ }^{21)}$
As one index of positioning accuracy, the standard deviation of positioning error in space depends on the PDOP, as expressed by Eq. (4),

$$
\sigma_{P}=P D O P \cdot \sigma_{U R E}
$$

where $\sigma_{U R E}$ is the standard deviation of range measurement error, and $\sigma_{P}$ is the standard deviation of three-dimensional space positioning error. ${ }^{22)}$ Accuracy and precision are used to evaluate the positioning performance of the system, and they represent the strength of system measurement error and random measurement error. Accuracy reflects the degree of accuracy in the measurement results with respect to the true value, and precision reflects the degree of stability in the positioning results. Some articles have introduced much information related to this field, and most studies were conducted before formal operation began in the Asian region. ${ }^{23-26)}$ By analyzing the results of these two indicators, we can conclude the accuracy of satellite positioning results. 3.1. $\mathrm{C} / \mathrm{N}_{\mathbf{0}}$

Figure 4 depicts the $\mathrm{C} / \mathrm{N}_{0}$ with elevation as estimated by the UR240-CORS receiver and UA240-CP antenna. The BeiDou satellites' B1 frequency and GPS L1 frequency C/ $\mathrm{N}_{0}$ values are depicted by the line of circles, and the BeiDou satellites' B2 frequency and GPS L2 frequency C/ $\mathrm{N}_{0}$ values are displayed as the line of pluses. The $\mathrm{L} 1 \mathrm{C} / \mathrm{N}_{0}$ of GPS Block-IIF and IIR satellites are larger than L2 by about 6-10 dB-Hz. However, BeiDou code observations of the B1 frequency have a significantly larger noise level than the code observations of the B2 frequency. Based on three orbits, the representative gap difference is $\mathrm{C} 01$ (GEO) of about $2-3 \mathrm{~dB}-\mathrm{Hz}$ and $\mathrm{C} 12$ (MEO) of about $2-4 \mathrm{~dB}-\mathrm{Hz}$. The gap of C06 decreases with elevation and B1 C/ $\mathrm{N}_{0}$ is close to $\mathrm{B} 2$ above 55 degrees, even stronger than B2. Comparing the $\mathrm{C} / \mathrm{N}_{0}$ values of the GPS and BeiDou MEO satellite signals, BeiDou $\mathrm{C} 12 \mathrm{~B} 2$ has a $\mathrm{C} / \mathrm{N}_{0}$ about $44-52 \mathrm{~dB}-\mathrm{Hz}$, while GPS $\mathrm{C} / \mathrm{N}_{0}$ values are lower than those of BeiDou. This is mainly due to the fact that the BeiDou GEO satellites have a higher level of signal power than other satellites.

As for GPS satellites, the $\mathrm{C} / \mathrm{N}_{0}$ for the $\mathrm{L} 2$ frequency starts at $30 \mathrm{~dB}-\mathrm{Hz}$, and it has a sharp increase with the increase in elevation, but it is also lower than the L1 frequency values. As shown in the figure, the $\mathrm{C} / \mathrm{N}_{0}$ values for the $\mathrm{L} 1$ frequency starts at about $40 \mathrm{~dB}-\mathrm{Hz}$, the $\mathrm{C} / \mathrm{N}_{0}$ value disparity between the L1 and L2 frequencies starts at about $10 \mathrm{~dB}$ -

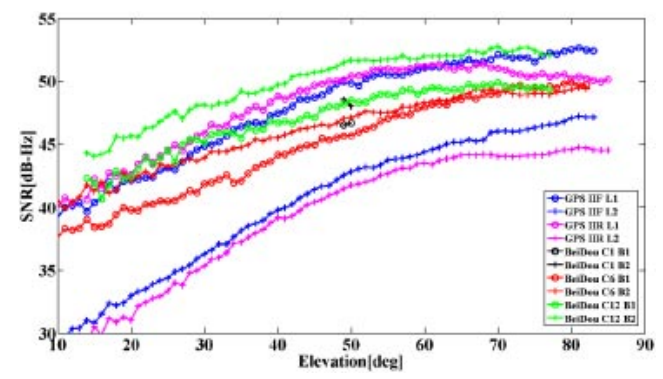

Fig. 4. $\mathrm{C} / \mathrm{N}_{0}$ of the UR240-CORS receiver and UA240-CP antenna for GPS and BeiDou satellites. 
$\mathrm{Hz}$, and there is about $8 \mathrm{~dB}-\mathrm{Hz}$ disparity at the elevation of 80 degrees.

As for BeiDou satellites, the $\mathrm{C} / \mathrm{N}_{0}$ values of the MEO satellites are larger than those of the GEO satellites at the same elevations, and the $\mathrm{C} / \mathrm{N}_{0}$ values of the GEO satellites are larger than those of the IGSO satellites. The $\mathrm{C} / \mathrm{N}_{0}$ value disparity decreases as elevation increases. The $\mathrm{C} / \mathrm{N}_{0}$ is defined as the ratio of the carrier signal power received to the noise power received, which indicates the quality of the signal received. So the larger $C / N_{0}$ value, the higher quality of the signal received.

\subsection{Multipath error}

Figure 5 (top/left) shows the time series of the B1 and B2 multipath for a typical BeiDou GEO C01 satellite. The elevation changes from 47 to 50 degrees. It can be seen that the complete time series of the B1 code multipath has larger variation than that of B2 codes, as the B1 code multipath error stays within $\pm 2.0 \mathrm{~m}$, while the $\mathrm{B} 2$ code multipath error stays within $\pm 1.0 \mathrm{~m}$. Figure 5 (top/right) shows the time series of the B1 and B2 multipath for a typical BeiDou IGSO C06 satellite. The elevation shows a "camelback" pattern and the cut-off elevation is set at 10 degrees, the top elevation is about 80 degrees. We can also see the IGSO C06 satellite skyplot in Fig. 3. The time series has a typical multipath effect during local time 8:00-16:00 due to the relatively low elevations. At the two highest elevations, the variations in multipath are relatively smaller. Figure 5 (mid/left) shows the time series of B1 and B2 multipath for a typical BeiDou MEO C11 satellite. The visible time length is about five hours from local time 17:00 to 22:00. The multipath effects change smoothly during the visible start time, but there are sharp variations at local time 21:00, and the value of multipath error rose to $20 \mathrm{~m}$. Figure 5 (mid/right) shows the time series for L1 and L2 multipath of a typical GPS G04 satellite for comparison. The observations were collected by the same receiver. As can be seen, the time series has typical multipath effects with stronger variations in meters between 17:0020:00 (local time), while it has a smaller multipath effect during the later time. The main difference is that the visible time length is only about eight hours. Figure 5 (bottom/left) shows the time series of L1 and L2 multipath for a typical GPS G11 satellite. The visible time length is about six hours. The time series has typical multipath effects with larger variations in meters at the start time within the required elevations, and there are smaller multipath effects during the later time. Figure 5 (bottom/right) shows the time series of L1 and L2 multipath for a typical GPS G16 satellite. The visible time length is about eight hours. There are smaller multipath effects near the higher elevations, but typical multipath effects at other times. In comparison, the BeiDou satellite multipath show smaller variations than GPS satellites. In Fig. 5, there are many discontinuities in multipath errors. This is because many discontinuities in carrier phase measurements occurred during analysis of the epochs.
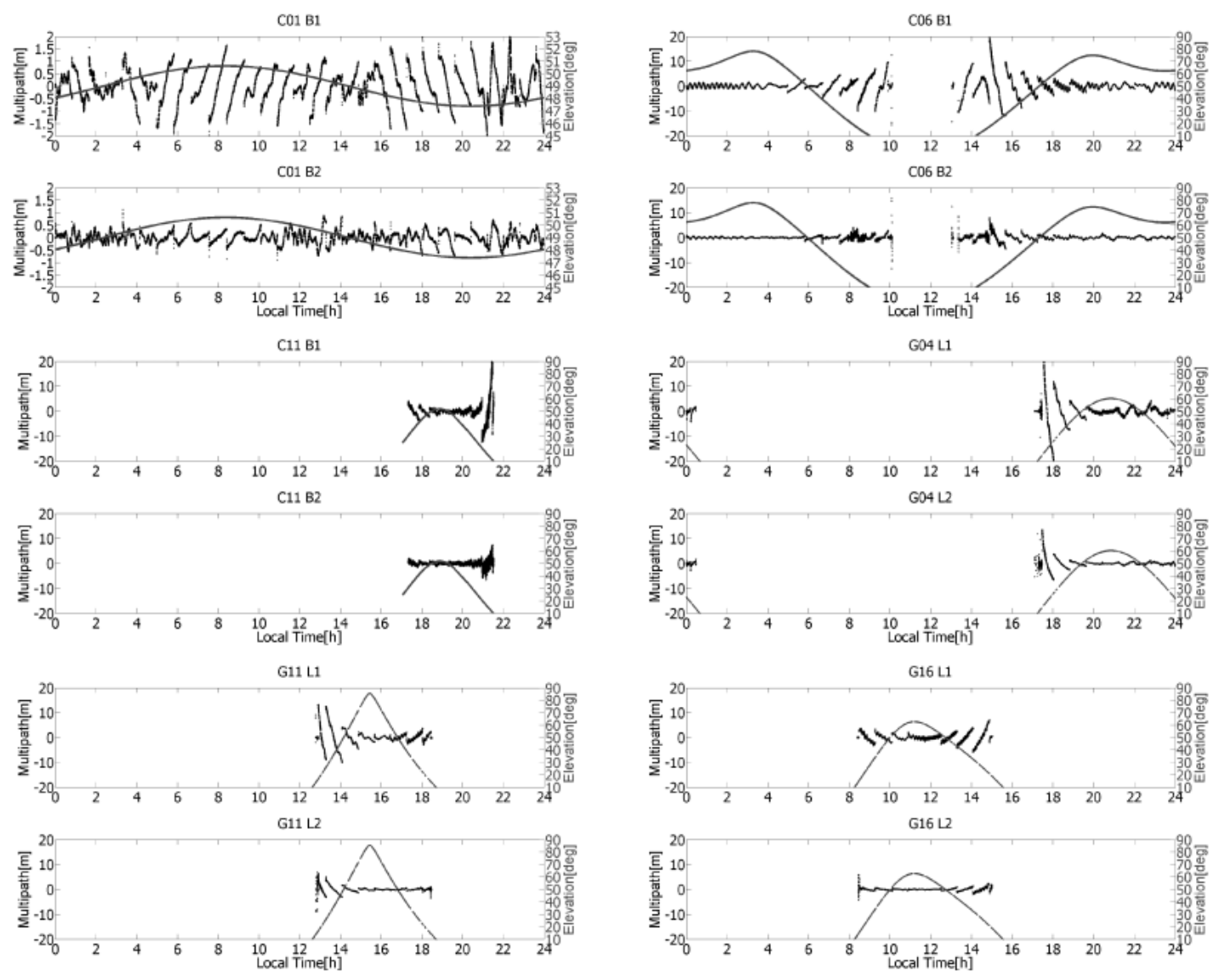

Fig. 5. B1/L1 and B2/L2 code multipath against the elevation for the BeiDou GEO C01 satellite (top/left), IGSO C06 satellite (top/right), MEO C11 satellite (mid/left), GPS G04 satellite (mid /right), GPS G11 satellite (bottom/left), and GPS G16 satellite (bottom/right). 
Table 3 lists variations in the root mean squares (RMS) of B1/L1 and B2/L2 multipath with elevations of 10-20 degrees, 40-50 degrees, and 70-80 degrees, using BeiDou C06 (IGSO), BeiDou C11 (MEO) and GPS G11 (MEO), respectively. Results indicate that the multipath errors of B1/ L1 are greater than B2/L2, BeiDou IGSO satellite multipath errors are greater than BeiDou MEO and GPS MEO satellite, and that BeiDou MEO satellite multipath errors are smaller than GPS MEO satellites.

\subsection{NVS}

Figure 6 depicts the NVS of the BeiDou system, GPS system and an integrated system on July 17, 2012 (top) and January 23, 2013 (bottom). The top figure shows that the NVS of the BeiDou system is less than that of the GPS system. However, after a half-year, the NVS of the BeiDou is more than that of the GPS from local time 7:00 to 21:00 (bottom figure). Since there are more visible satellites after using BeiDou's GEO and IGSO, NVS is more stable than GPS. The NVS is greatly improved in the integrated system. This means that there are more opportunities to choose high-quality satellites to achieve good positioning performance. The integrated system provides better validity for positioning.

\subsection{PDOP}

Figure 7 displays the PDOP variation in NVS for the GPS/BeiDou integrated system (top), GPS (middle) and BeiDou system (bottom) on July 17, 2012 and January 23, 2013. The PDOP is depicted by the black line and the NVS is depicted by the gray line. As the NVS of the integrated system contains BeiDou and GPS for all visible satellites, the PDOP value is maintained between 1 and 2 . The PDOP of the integrated system decreases 1 to 2 , representing

Table 3. The variations in RMSs of $\mathrm{B} 1 / \mathrm{L} 1$ and $\mathrm{B} 2 / \mathrm{L} 2$ multipath with elevations of 10-20 degrees, 40-50 degrees, and 70-80 degrees.

\begin{tabular}{cccc}
\hline $\begin{array}{c}\text { Elevation } \\
\text { coverage } \\
\text { (degree) }\end{array}$ & $\begin{array}{c}\text { Satellite } \\
\text { number }\end{array}$ & $\begin{array}{c}\text { B1/L1 } \\
\text { Standard deviation } \\
(\mathrm{m})\end{array}$ & $\begin{array}{c}\text { B2/L2 } \\
\text { Standard deviation } \\
(\mathrm{m})\end{array}$ \\
\hline 10-20 & BeiDou C06 & 2.452 & 2.025 \\
& BeiDou C11 & $\mathbf{2 . 0 1 8}$ & $\mathbf{1 . 6 3 9}$ \\
& GPS G11 & 2.029 & 1.657 \\
\hline $40-50$ & BeiDou C06 & 0.327 & 0.281 \\
& BeiDou C11 & $\mathbf{0 . 2 8 3}$ & $\mathbf{0 . 2 5 9}$ \\
& GPS G11 & 0.307 & 0.270 \\
\hline $70-80$ & BeiDou C06 & 0.243 & 0.179 \\
& BeiDou C11 & $\mathbf{0 . 1 9 4}$ & $\mathbf{0 . 1 1 8}$ \\
& GPS G11 & 0.218 & 0.131 \\
\hline
\end{tabular}

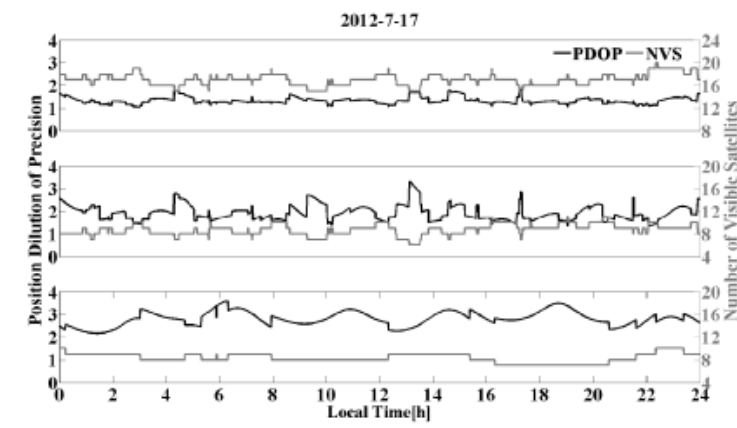

that it is smaller and more stable than a single system. This means that the integrated system may have more options for choosing high-quality satellites to obtain more accurate positioning results, and higher reliability to ensure positioning in harsh environments.

The NVS of the BeiDou system is larger than that of the GPS system in Fig. 6 (right) from local time 7:00 to 21:00, while PDOP doesn't exhibit the corresponding changes that all values above 2 are worse than GPS system. Meanwhile, relative to the GPS system, the PDOP of the BeiDou system is more stable. This phenomenon may be related to the BeiDou space layout, where five GEO satellites stationary relative to the Earth are always visible from Shanghai.

\subsection{Position accuracy}

In our experiment, to detect BeiDou positioning accuracy changes, several days' observation data were prepared for this experiment during July 2012 (before formal regional operation) and January 2013 (after formal regional operation). All positioning results were estimated by single-point positioning. Accuracy was calculated by subtracting the measurement results from the actual location, and precision was calculated using RMS of position results.

Figure 8 displays the horizontal deviation and vertical deviation relative to actual position using the GPS, BeiDou and integrated systems on July 16, 2012 and January 21, 2013. The thick black line represents deviation using the integrated system and the two gray lines show the deviation of the BeiDou and GPS systems. One thick light gray line represents

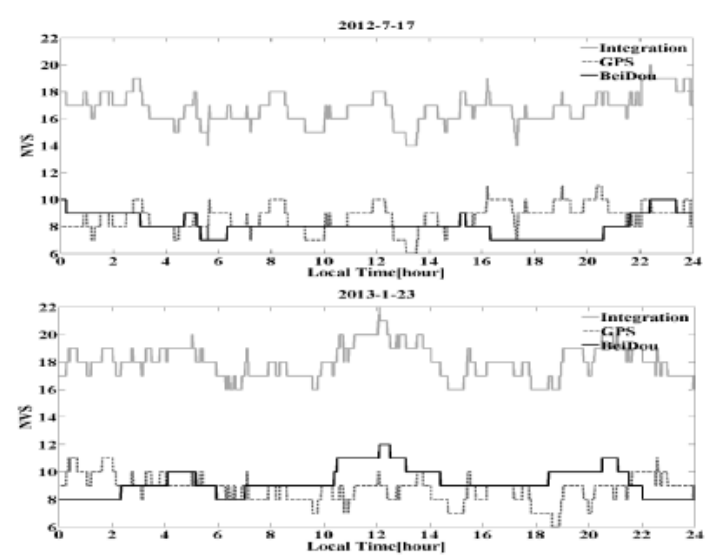

Fig. 6. NVS for the BeiDou, GPS and integrated systems on July 17, 2012 (top) and January 23, 2013 (bottom).

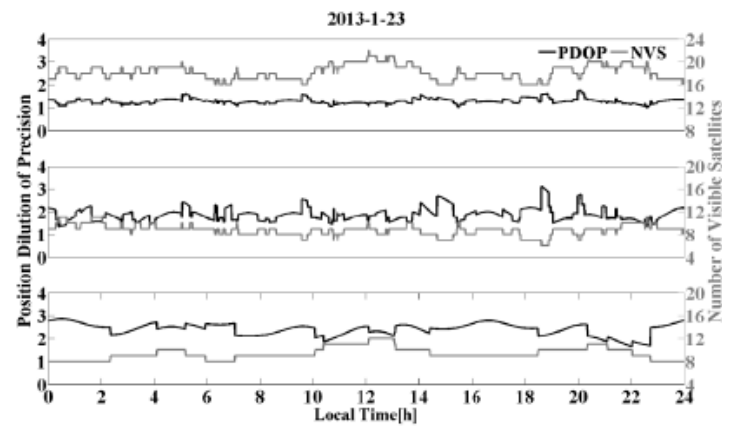

Fig. 7. PDOP and NVS for the integrated system (top), GPS only (middle), BeiDou only (bottom) on July 17, 2012 (left) and January 23 , 2013 (right). 

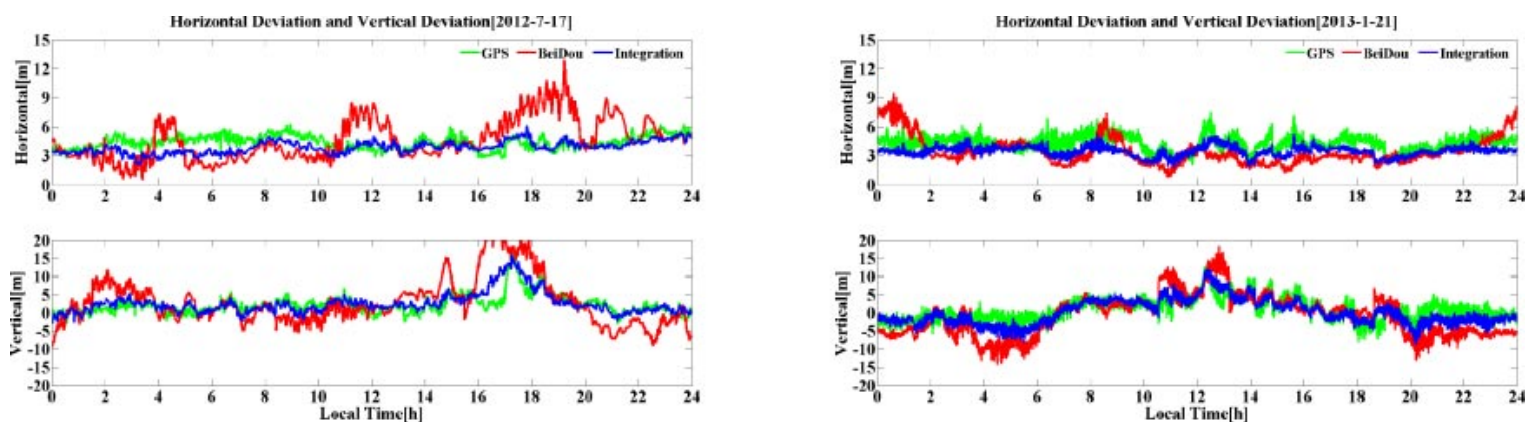

Fig. 8. Horizontal deviation (top) and vertical deviation (bottom) (relative to actual position) on July 17, 2012 (left) and January 21, 2013 (right).

Table 4. The variations in RMS on July 17 and 19, 2012, and January 21 and 23, 2013.

\begin{tabular}{|c|c|c|c|c|c|c|c|c|}
\hline \multirow{3}{*}{ System } & \multicolumn{4}{|c|}{$\begin{array}{l}\text { Before formal regional } \\
\text { operation }\end{array}$} & \multicolumn{4}{|c|}{$\begin{array}{l}\text { After formal regional } \\
\text { operation }\end{array}$} \\
\hline & \multirow{2}{*}{ Date } & \multicolumn{3}{|c|}{ RMS (m) } & \multirow{2}{*}{ Date } & \multicolumn{3}{|c|}{ RMS (m) } \\
\hline & & Lon. & Lat. & $\mathrm{H}$ & & Lon. & Lat. & $\mathrm{H}$ \\
\hline$\overline{\mathrm{GPS}} / \mathrm{t}$ & $2012-7-17$ & 0.77 & 1.75 & 2.54 & $2013-1-21$ & 0.45 & 1.70 & 2.27 \\
\hline GPS & & 1.22 & 1.73 & 3.15 & & 0.56 & 2.36 & 3.1 \\
\hline BeiDou & & 1.96 & 3.36 & 5.90 & & 0.80 & 2.17 & 5.62 \\
\hline GPS/Beil & $2012-7-19$ & 0.89 & 0.82 & 1.88 & $2013-1-23$ & 0.50 & 1.52 & 1.5 \\
\hline GPS & & 1.29 & 1.23 & 2.71 & & 0.74 & 1.86 & 2.6 \\
\hline BeiDou & & 2.37 & 2.82 & 5.73 & & 0.58 & 1.91 & 4.0 \\
\hline
\end{tabular}

the GPS and the thin dark gray line is the BeiDou system. As is shown in the figure (left), the positioning accuracy value of integrated system has less than $5 \mathrm{~m}$ deviation horizontally and less than $10 \mathrm{~m}$ deviation vertically. The GPS system has a slightly higher value than the integrated system, but has better accuracy performance than the BeiDou system. The positioning accuracy value of the BeiDou system is under $15 \mathrm{~m}$ horizontally, and less than $20 \mathrm{~m}$ vertically.

The positioning performance using the BeiDou system on January 21, 2013 shows more stability and accuracy than on July 16, 2012. The BeiDou is lower than $10 \mathrm{~m}$, close to the GPS system horizontally, and vertical positioning performance is close to the GPS system. The performance on January 21, 2013 matches the accuracy reported by the Chinese State Council Information Office.

Table 4 lists the variations in RMS on July 17 and 19, 2012 and January 21 and 23, 2013 in respective directions. After formal regional operation using the BeiDou system, the RMS of the system is almost the same as the GPS system in the horizontal domain, and also larger than the GPS system in the height domain. This means that BeiDou positioning performance is close to that of GPS, and also not stable relative to GPS in the height domain. After integrating the BeiDou and GPS systems, the accuracy of the integrated system was obviously improved following formal regional operation of the BeiDou system, especially in the height domain.

\subsection{Data analysis in an urban environment}

In order to compare the positioning performance in different places, we also carried out an experiment in the urban environment on January 26, 2013 from 17:37 to 17:54 (local time). The data we collected was for about 15 minutes. The same UR240-CORS GPS/BeiDou quad-frequency

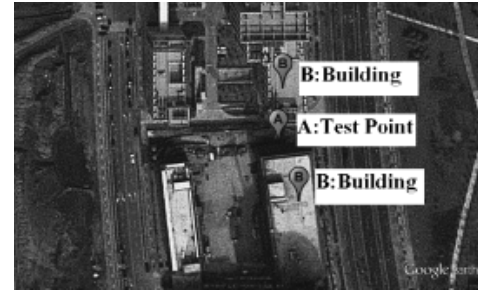

Fig. 9. Test point in an urban environment on January 26, 2013 (from Google Earth).

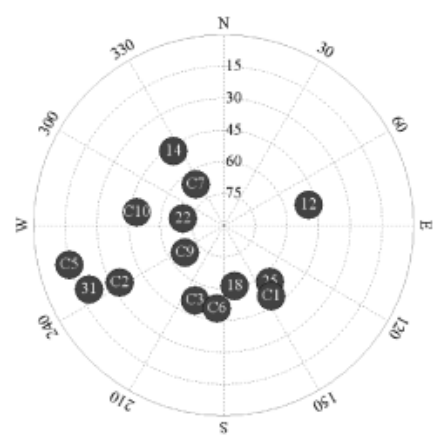

Fig. 10. GSP/BeiDou skyplot in an urban environment at 17:37 (local time) on January 26, 2013.

high-precision receiver was used for this experiment, and a UA240-CP GPS/BeiDou quad-frequency, high-gain antenna was also used to support the receiver. There were high buildings on the north and south sides and open on east and west sides. Figure 9 shows the experiment environment. In Fig. 9, label "A" represents the test point and label "B" represents buildings. In order to see the distribution of satellites clearly, Fig. 10 depicts the skyplot of the satellites: C01 to $\mathrm{C} 14$ represent BeiDou satellites and the others are GPS satellites. From the skyplot it can be seen that the BeiDou system has more visible satellites than the GPS system, especially on the north and south sides of the test point. Because the BeiDou system has high-elevation satellites susch as GEO and IGSO, buildings have less positioning influence on the BeiDou system than on the GPS system. Table 5 shows the comparison results between different systems. The results analysis included the available epoch, PDOP and RMS. When the PDOP is less than 30 and the NVS is more than 4 at a certain epoch, it is considered that visibility is possible at that epoch. In our experiments, we considered 
Table 5. Comparisons of available epoch, PDOP and RMS between different systems.

\begin{tabular}{|c|c|c|c|c|c|c|c|}
\hline \multirow{2}{*}{$\begin{array}{l}\text { Mask } \\
\text { angle }\end{array}$} & \multirow{2}{*}{ System } & \multirow{2}{*}{$\begin{array}{c}\begin{array}{c}\text { Available } \\
\text { epochs }\end{array} \\
(\mathrm{PDOP} \leq 30 \& \\
\mathrm{NVS} \geq 4)\end{array}$} & \multicolumn{2}{|c|}{ RMS (m) } & \multicolumn{3}{|c|}{$\operatorname{PDOP}(\leq 30)$} \\
\hline & & & Lon. & Lat. $\mathrm{H}$ & Max. & Min. & Mean \\
\hline \multirow[t]{3}{*}{5 degrees } & $\begin{array}{l}\text { GPS/ } \\
\text { BeiDou }\end{array}$ & $964(100 \%)$ & 3.79 & 0.541 .59 & 2.45 & 2.05 & 2.20 \\
\hline & GPS & 867 (90\%) & 5.09 & $1.27 \quad 2.19$ & 3.40 & 2.94 & 3.22 \\
\hline & BeiDou & 964 (100\%) & 4.41 & 1.544 .47 & 5.35 & 3.47 & 3.99 \\
\hline
\end{tabular}

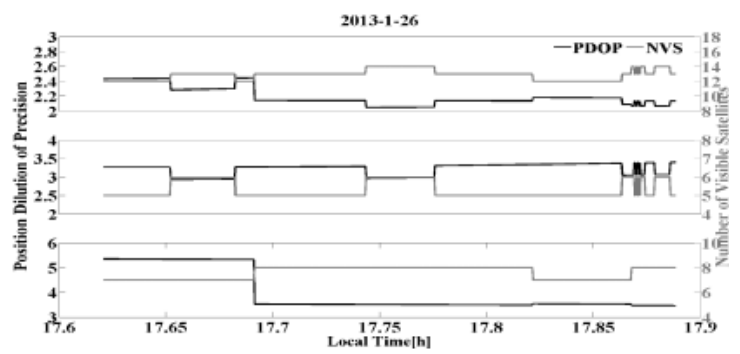

Fig. 11. PDOP and NVS for the integration system (top), GPS only (middle), BeiDou only (bottom) on January 26, 2013.

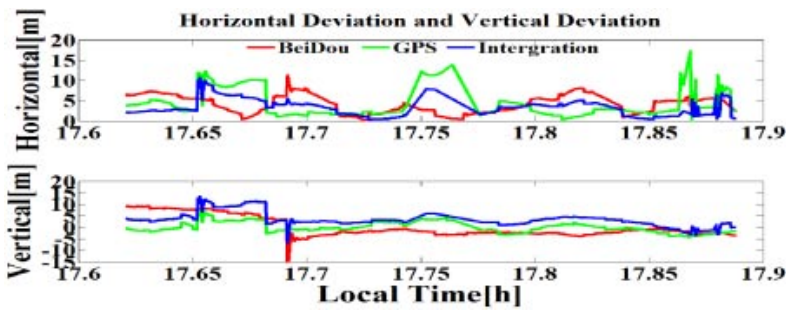

Fig. 12. Horizontal deviation (top) and vertical deviation (bottom) on January 26, 2013.

that positioning was effective only when the PDOP was less than 30 at a certain epoch. The precision is also calculated using the RMS of position results. But, in fact, we do not know the actual location of the test post, so we replaced actual locations with average results.

Figure 11 displays the PDOP variation with NVS for the GPS/BeiDou integrated system (top), and GPS (middle) and BeiDou (bottom) systems on January 26, 2013. Figure 12 displays horizontal deviation and vertical deviation using the GPS, BeiDou and integrated systems on January 26, 2013. From Table 4, it can be seen that the available epochs of the BeiDou and GPS/BeiDou systems are 964, and the percentage is $100 \%$, which represents that all epochs are available. However, available epochs of the GPS system are 867 and the percentage is $90 \%$, which represents that $10 \%$ of the epochs are not available. The availability can be improved using the BeiDou system only or by integrating the GPS/BeiDou systems. The RMS of the BeiDou system is better than the GPS system in the longitude domain, but in the latitude domain and height domain the BeiDou system is worse than the GPS system. The mean PDOP of the BeiDou system is also worse than the GPS system. After integrating the BeiDou and GPS systems, the RMS of positioning results was greatly improved not only in the horizontal domain, but also in the height domain. The PDOP can also be improved. The positioning performance after using the BeiDou system can be evaluated under an urban environment in Shanghai.

\section{Conclusion}

In this paper, the positioning performance using a BeiDou system, a GPS system and an integrated system was compared. We first gave the plots of $\mathrm{C} / \mathrm{N}_{0}$. The BeiDou system possesses three kinds of characteristics depending on different orbits. Comparing the MEO C/ $\mathrm{N}_{0}$ value of the BeiDou and GPS systems, BeiDou C12 B2 had C/ $\mathrm{N}_{0}$ values of about 44-52 dB-Hz, while the GPS $\mathrm{C} / \mathrm{N}_{0}$ values were lower than those of the BeiDou system. In a multipath error comparison, the BeiDou satellites multipath showed small variations than GPS satellites.

In results analysis, three indicators (e.g., NVS, PDOP, accuracy and precision) were used to assess the performance of the system. The results show that the NVS of the BeiDou system was more than that of the GPS system from 7:00 to 21:00 (local time) on January 23, 2013, and the integrated system had the largest NVS, lowest PDOP value, and best value for accuracy and precision. The PDOP of the integrated system can be decreased 1-2, representing smaller deviation and better stability than single systems. The RMS of the integrated system can be improved about $35 \%$ in the longitude domain, $23 \%$ in the latitude domain, and $32 \%$ in the height domain. Comparing BeiDou system positioning results between before and after formal regional operation, the RMS can be improved about $68 \%$ in the longitude domain, $34 \%$ in the latitude domain, and $17 \%$ in the height domain after formal regional operation. The constellation adjustment impact, especially in the horizontal domain, can be seen. In an urban environment, the integrated system also has the lowest PDOP value, and the smallest RMS of the positioning results. The RMS can be improved about $25 \%$ in the longitude domain, $57 \%$ in the latitude domain, and $27 \%$ in the height domain. The GPS system showed better positioning than the BeiDou system with smaller deviation. However, because of BeiDou's GEO and IGSO satellites, more highelevation satellites can be seen, and the available epochs can be improved about $11 \%$ by using the BeiDou system.

After six month of adjustment, the BeiDou system can meet the accuracy requirements, which is a positioning accuracy of less than $10 \mathrm{~m}$. Especially in the horizontal domain, its positioning performance improved significantly, becoming close to the GPS system. However, in the height domain, the positioning performance is worse than that of the GPS system. The BeiDou system can also improve the availability of positioning, especially in urban environments. Since there are five GEO stationary satellites relative to the Earth in the BeiDou system, the PDOP of the BeiDou system is currently poorer than the GPS system. As more BeiDou MEO satellites are launched in the future, it will not only improve PDOP and positioning accuracy, but also improve the stability of the integrated system. 


\section{Acknowledgments}

This research was supported by the National Natural Science Foundation of China (Grant No. 41376178, 41401489), the Program for Professor of Special Appointment (Eastern Scholar) at Shanghai Institutions of Higher Learning, Shanghai Science and Technology Committee (Project 11510501300), Shanghai Foundation for University Youth Scholars (ZZHY13033), and special funding for the development of science and technology of Shanghai Ocean University (A2-0209-14-200070). The authors would like to thank Mr. Tomoji Takasu, Tokyo University of Marine Science and Technology, Japan, and Dr. Falin Wu, Beihang University, for their discussion on position performance evaluation. The authors would also like to thank Dr. Zhonghua Hong, Shanghai Ocean University, for his checking and correcting English of this article.

\section{References}

1) De Wilde, W., Boon, F., Sleewaegen, J. M., and Wilms, F.: More Compass Points-Tracking China's MEO Satellite on a Hardware Receiver, Inside GNSS, 2, 5 (2007), pp. 44-48.

2) Ran, C.: COMPASS Satellite System Development and Plan, The First China Satellite Navigation Conference, Beijing, 2010.

3) Ran, C.: BeiDou ICD Released, GPS World, 2013.

4) Shi, C., Zhao, Q., Hu, Z., and Liu, J.: Precise Relative Positioning using Real Tracking Data from COMPASS GEO and IGSO Satellites, GPS Solut., 2011, DOI: 10.1007/s10291-012-0264-x

5) Montenbruck, O., Hauschild, A., Steigenberger, P., Hugentobler, U., Teunissen, P., and Nakamura, S.: Initial Assessment of the COMPASS/BeiDou-2 Regional Navigation Satellite System, GPS Solut., 2012, DOI: 10.1007/s10291-012-0272-X

6) Jin, R., Jin, T., and Feng, G.: M_DCB: Matlab Code for Estimating GNSS Satellite and Receiver Differential Code Biases, GPS Solut., 2012, DOI: 10.1007/s10291-012-0279-3

7) Zhang, Y.: Performance Analysis of GPS Augmentation Using QuasiZenith Satellite System in Local Japan Area, ION GNSS 19th International Technical Meeting of the Satellite Division, Fort Worth, USA, 2006.

8) Zhang, Y., Wu, F., and Yasuda, A.: Impact of Integrated GPS and the Quasi-Zenith Satellite System in the East Asian Region, T. Japan Soc. Aeronaut. Space Sci., 50 (2007), pp. 105-112.

9) Greilier, T., Dantepal, J., Delatour, A., Ghion, A., and Enge, P.: Initial Observations and Analysis of COMPASS MEO Satellite Signal, Inside GNSS, 2, 4 (2007), pp. 39-43.

10) Fan, L. and Chai, H.: Study on Method of Analyzing the Positioning Accuracy of Beidou 2nd Generation Satellite Navigation System, $\mathrm{Hy}$ drographic Surveying and Charting, 29, 1 (2009), pp. 25-27.

11) Wang, M., Sun, G., Wang, F., and Yong, S.: Weighted Geometric Di- lution of Precision's Analysis for Mixed Constellation Navigation System, Chinese Space Science and Technology, 27, 5 (2007\}, pp. 50-56.

12) Mertikas, S., Wells, D. E., and Leenhouts, P.: Treatment of Navigational Accuracies: Proposals for the Future. Navigation, J. Inst. Navigation, 32, 1 (1985), pp. 68-84.

13) Wolf, R.: Satellite Orbit and Ephemeris Determination using Inter Satellite Link, Ph.D. Thesis, University of Bundeswedr, Munchen, Germany, 2000.

14) Chen, M., Zhang, P., and Sun, Z.: GPS Multipath Error Analysis and Detection Methods, Engineering of Surveying and Mapping, 20, 5 (2011), pp. 29-35.

15) Yin, H. and Xiong, Y.: The Study on Multipath Effect of GPS Surveying, Railway Air Survey, 2003, 3 (2003), pp. 12-14.

16) Chang, S., Yang, G., Lin, P., and Wu, J.: An Analysis on Combined GPS/COMPASS Data Quality and Its Effects on Single Point Positioning Accuracy under Different Observing Conditions, Advances in Space Research, 54, 5 (2013), pp. 818-829.

17) Satirapod, C. and Wang, J.: Comparing the Quality Indicators of GPS Carrier Phase Observations, Geomatics Research Australasia, 73 (2000), pp. 75-92.

18) Kee, C. and Parkinson, B.: Calibration of Multipath Errors on GPS Pseudorange Measurements, Proceedings of the 7th International Technical Meeting of the Satellite Division of Institute of Navigation (ION GPS 1994), Salt Lake City, UT, 1994, pp. 353-362.

19) Hauschild, A., Steigenberger, P., and Rodriguez-Solano, C.: Signal, Orbit and Attitude Analysis of Japan's First QZSS Satellite Michibiki, GPS Solut., 2011, DOI: 10.1007/s10291-011-0245-5

20) Zhao, W., Ren, H., and Li, M.: Analysis of the Multipath Impact on Dual-Frequency Positioning Error under GEO and IGSO Constellation, The Third China Satellites Navigation Conference, Guangzhou, 2012.

21) Zhang, Y., Kubo, N., and Yasuda, A.: The Benefit from Triple Frequencies in Ambiguity Resolution, J. Japan Inst. Navigation, 112 (2005), pp. 219-227.

22) Kaplan, E. D. and Hegarty, C. J.: Understanding GPS Principles and Applications, Artech House Publishers, 2005.

23) Chen, L., Zhao, Q., Hu, Z., Zhao, Y., and Xiang, F.: Preliminary Analysis on Pseudorange Data Quality and Positioning Accuracy of BeiDou Satellites Navigation System, The Third China Satellites Navigation Conference, Guangzhou, 2012.

24) Chen, W., Gao, C., and Wu, Y.: Study on Static Differential Positioning Based on GPS/Compass Combined System, The Third China Satellites Navigation Conference, Guangzhou, 2012.

25) Li, M.: Precise Point Positioning with BeiDou Navigation Satellites System, The Third China Satellites Navigation Conference, Guangzhou, 2012.

26) Gao, W., Su, M., and Li, C.: An Evaluation of BeiDou Navigation Satellite System, The Third China Satellites Navigation Conference, Guangzhou, 2012. 